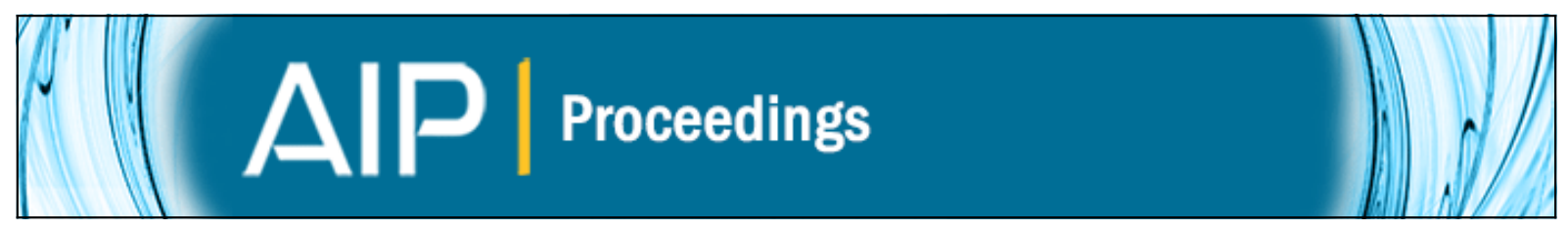

\title{
Seismology of White Dwarfs: The ZZ Ceti Stars
}

Barbara G. Castanheira and S. O. Kepler

Citation: AIP Conference Proceedings 1170, 616 (2009); doi: 10.1063/1.3246572

View online: http://dx.doi.org/10.1063/1.3246572

View Table of Contents: http://scitation.aip.org/content/aip/proceeding/aipcp/1170?ver=pdfcov

Published by the AIP Publishing

Articles you may be interested in

The ZZ Ceti star HS 0507+0434B revisited

AIP Conf. Proc. 1273, 516 (2010); 10.1063/1.3527878

Discovery of twelve ZZ Ceti stars

AIP Conf. Proc. 1273, 500 (2010); 10.1063/1.3527874

Light Curve Patterns and Seismology of a White Dwarf with Complex Pulsation

AIP Conf. Proc. 1170, 625 (2009); 10.1063/1.3246574

White Dwarf and Pre-White Dwarf Pulsations

AIP Conf. Proc. 1170, 605 (2009); 10.1063/1.3246571

Magnetic Accretion Onto White Dwarfs

AIP Conf. Proc. 797, 257 (2005); 10.1063/1.2130241 


\title{
Seismology of White Dwarfs: The ZZ Ceti Stars
}

\author{
Barbara G. Castanheira* and S. O. Kepler ${ }^{\dagger}$ \\ ${ }^{*}$ Department of Astronomy and McDonald Observatory, University of Texas, Austin, TX 78712, USA \\ ${ }^{\dagger}$ Departamento de Astronomia, Universidade Federal do Rio Grande do Sul, Av. Bento Gon Ãğalves 9500 Porto \\ Alegre 91501-970, RS, Brazil
}

\begin{abstract}
We calculate an extensive adiabatic model grid for pulsating white dwarfs with $\mathrm{H}$ dominated atmospheres, the ZZ Ceti stars. We developed a new approach for asteroseismology, using the relative observed amplitudes as weights, and compared the computed modes with the observed ones for the class of ZZ Ceti stars. We measure the H layer mass for 83 stars and found an average of $M_{H} / M_{*}=10^{-6.3}$, which is thinner than the predicted value of $M_{H} / M_{*}=10^{-4}$. Our results indicate that the stars lose more mass during their evolution than previously expected.
\end{abstract}

Keywords: Pulsations, Oscillations, and Stellar Seismology

PACS: $97.10 . \mathrm{Sj}$

\section{INTRODUCTION}

White dwarfs, the final evolutionary stage of $\sim 95 \%$ of all stars (e.g., [12]), have their evolution dominated by cooling. As they cool, because of the $\kappa-\gamma$ mechanism and/or convection driving, they pulsate in different instability strips, depending on the chemical element that drives pulsation. The DAVs or ZZ Ceti stars have hydrogen atmospheres and are confined in an observational instability strip between 10800 and $12300 \mathrm{~K}$. Recent observations $[8,21]$ indicate that pulsation is an evolutionary phase for white dwarfs. Therefore, properties measured for pulsators can potentially be applied to all white dwarfs.

Asteroseismology is a powerful tool to probe the internal structure of the stars, as every pulsation mode is an independent measurement. Even from just a few modes, we can estimate fundamental stellar properties [9]. When a large number of modes is observed, it is possible to study the internal stellar structure in detail (e.g., [31]).

The ZZ Ceti stars can be separated in three groups: hot, intermediate, and cool. The hot ZZ Ceti stars have only a few excited modes, with short periods $(P<300 \mathrm{~s})$, small amplitudes $(1.5<A<20 \mathrm{mma})$, and the power spectrum does not change substantially from season to season, and they define the blue edge of this instability strip. The cool ZZ Ceti stars, located in the red edge, show a very rich pulsational spectra of high amplitude modes (up to $30 \%$ ) and longer periods (up to $1500 \mathrm{~s}$ ). The modes interfere with each other, causing dramatic changes in the pulsational spectra. A few red edge pulsators have long periods of low amplitude, representing the stage when stars stop to pulsate. The intermediate ZZ Ceti stars have characteristics between the hot and the cool groups.

\section{THE SEISMOLOGICAL MODELS}

As the internal structure of white dwarfs with $T_{\text {eff }} \sim$ $12000 \mathrm{~K}$ has been sufficiently modified due to cooling, stellar contraction, and chemical diffusion, a hot polytrope static model is equivalent to self-consistent models (e.g. [38]).

We used the White Dwarf Evolutionary Code $[28,38]$ to evolve the starting model until the desired temperature, with distinct equation of states for the core [27] and for the envelopes [11]. We used the updated opacity OPAL tables [15], neutrino rates [16], and ML $2 / \alpha=0.6$ MLT [4]. The core evolution calculations are selfconsistent, but the envelope was treated separately. The core and the envelope are stitched together, fulfilling the boundary conditions in the interface. The transitions between the layers are consistent with time-dependent diffusion [1].

We calculated an extensive adiabatic model grid for pulsation modes, varying $T_{\mathrm{eff}}, M, M_{\mathrm{H}}$, and $M_{\mathrm{He}}$. The grid was generated from a starting polytrope of $M=0.6 M_{\odot}$, using homology transformations to obtain models with masses from 0.5 to $1.0 M_{\odot}$, in steps of $0.005 M_{\odot}$. The effective temperature ( $\left(T_{\mathrm{eff}}\right)$ varies from 10600 to $12600 \mathrm{~K}$, in steps of $50 \mathrm{~K}$. The upper $\mathrm{H}$ and He layer masses are $10^{-4} M_{*}$ and $10^{-2} M_{*}$, respectively; above these limits, nuclear reactions would excite $g$-mode pulsations in the PNNVs by the $\varepsilon$ mechanism, which are not observed [14]. The lower $\mathrm{H}$ and $\mathrm{He}$ layer mass values are $10^{-9.5} M_{*}$ (the minimum $\mathrm{H}$ amount to be detected in a DA white dwarf spectra) and $10^{-3.5} M_{*}$, respectively.

Varying the core composition introduces three parameters in the fit: the abundance itself from the uncertain $\mathrm{C}(\alpha, \gamma) \mathrm{O}$ reaction rate [31] and the two extreme points of the function that better describes the transition zone. We chose a homogeneous core of 50:50 C/O, for sim- 
plicity. For a model with $T_{\text {eff }}=11600 \mathrm{~K}, M=0.6 M_{\odot}$, $M_{\mathrm{H}}=10^{-4} M_{*}$, and $M_{\mathrm{He}}=10^{-2} M_{*}$, the change for the three first $\ell=1$ overtones is only a few seconds when we vary the $\mathrm{O}$ core abundance from $0 \%$ to $90 \%$ ( $\mathrm{C}$ is the remainder quantity).

The transition zone between the core and the He outer envelope for a Salaris-like profile [36] is in a different position than the models we used, causing the trapped modes in these cavities to be different. Because the $Z Z$ Ceti stars pulsate with only a few modes, we decided to use a fixed homogeneous C/O 50:50 core to decrease the number of free parameters, but still be consistent with the reaction rate uncertainty. The price paid for our choice is that the He layer mass determinations are uncertain, as the differences in the shape of the transition zone can be compensated by changes in the thickness of the He layer. The Salaris profile introduces a more complicated than a linear decrease of the $O$ profile in the outer layers (see Fig. 2. of [31]).

\section{G117-B15A: OUR TEST PARTICLE}

Since the discovery of its variability [30], G117-B15A was considered the most regular $Z Z$ Ceti. Nowadays, G117-B15A is the most precise optical clock known, with measured rate of change of the main mode with a precision better than $10^{-15} \mathrm{~S} / \mathrm{s}$ [19].

G117-B15A pulsates with three independent low amplitude modes, showing the harmonic of the main mode and linear combinations between modes [17]. In Table 1, we list the average values for all periodicities detected in observations with the $2.1 \mathrm{~m}$ telescope at McDonald Observatory.

TABLE 1. All detected periodicities (modes, linear combinations, and harmonics) for G117-B15A.

\begin{tabular}{ccc}
\hline Period (s) & Amplitude (mma) & identification \\
\hline 215.20 & 17.36 & $\mathrm{f} 1$ \\
270.46 & 6.14 & $\mathrm{f} 2$ \\
304.05 & 7.48 & $\mathrm{f} 3$ \\
\hline 107.7 & 1.65 & $2 \times \mathrm{f} 1$ \\
126.2 & 1.40 & $\mathrm{f} 1+\mathrm{f} 3$ \\
119.8 & 1.30 & $\mathrm{f} 1+\mathrm{f} 2$ \\
\hline
\end{tabular}

We used G117-B15A as a test of our seismological analysis because it shows a simple pulsation spectrum, but more than one mode is excited, and there are independent seismological studies, which we used to test our approach.

Our seismological study starts with a literature search for all available information about a star. Besides more than 30 years of time series photometry [19], there are independent measurements of atmospheric properties ( $T_{\text {eff }}$ and $\log g$ ) for G117-B15A, from different techniques $[3,24,37]$.

As our models do not account for the excitation of linear combinations nor harmonics, it is very important to be sure that only normal modes are being used in the fit. For G117-B15A, the modes are identified in Table 1 as $f_{1}, f_{2}$, and $f_{3}$. We then compared the observed modes $\left(P_{\text {obs }}\right)$ with the computed ones $\left(P_{\text {model }}\right)$ by adding the square of the differences. We choose to weight the periods by pulsation energy, which is proportional to the observed amplitude squared $\left(w_{P} \propto A^{2}\right)$, minimizing $S$ :

$$
S=\sum_{i=1}^{n} \sqrt{\frac{\left[P_{\mathrm{obs}}(i)-P_{\mathrm{model}}\right]^{2} \times w_{P}(i)}{\sum_{i=1}^{n} w_{P}(i)}}
$$

where $n$ is the number of observed modes and $w_{P}$ is the weight given to each mode.

Even though our model grid uses the adiabatic approximation and does not provide amplitudes, our choice to weight our fits is to guarantee that the fit will be dominated by high amplitude modes. For G117-B15A, the periods and their respective normalized weights are $215.20 \mathrm{~s}, 270.46 \mathrm{~s}$, and $304.05 \mathrm{~s}$, and $0.13,1.00$, and 0.67 .

At first, we try $\ell=1$ for all modes, supported by previous observations $[35,26]$. We would have tried higher $\ell$ values if no solution was found, despite that all chromatic amplitude changes to date indicate the modes are more likely $\ell=1$ for all studied ZZ Ceti stars (e.g., [18]).

By comparing the observations and the models, we found several families of solution below the cut $S<1.8 \mathrm{~s}$ (the quadratic sum of the relative uncertainties). Figure 1 shows the possible combinations of $T_{\mathrm{eff}}, M, M_{\mathrm{H}}$, and $M_{\mathrm{He}}$ below the $S$ cut off, and the minimum of each family is listed in Table 2.

The mean uncertainties for the quantities we fit are $\sigma_{T_{\text {eff }}} \sim 50 \mathrm{~K}, \sigma_{M} \sim 0.005 M_{\odot}, \sigma_{M_{\mathrm{H}}} \sim 10^{-0.5} M_{*}$, and $\sigma_{M_{\mathrm{He}}} \sim 10^{-0.5} M_{*}$, of the order of the spacings in the model grid. These values are the typical uncertainties in our seismological studies.

The final step is to identify which of the possible solutions are consistent with independent atmospheric determinations. From previous determinations, $0.46<$ $M / M_{\odot}<0.66$ and $11430 \mathrm{~K}<T_{\text {eff }}<12040 \mathrm{~K}$, which leaves only the first solution in Table 2 as a possibility. Our seismological solution indicates that $T_{\text {eff }}$ is hotter than derived from the optical spectra [3], but consistent UV spectroscopy [24].

Alternatively to our procedure, Bradley [5] selects seismological models consistent with spectroscopic mass and temperature. He uses a standard model with $M_{\mathrm{He}}=$ $10^{-2} M_{*}$ and $\mathrm{C} / \mathrm{O}$ nominal profile. Afterwards, he adjusts $M_{\mathrm{H}}$ to obtain a mode with $\ell=1, k=1$ or 2 close to $215 \mathrm{~s}$. Then, he refines $T_{\text {eff }}$ and $M_{\mathrm{H}}$ to bring the $270 \mathrm{~s}$ mode into agreement and uses the $304 \mathrm{~s}$ mode to adjust the 
TABLE 2. Seismological solutions for G117-B15A: absolute minima for each possible family of solution in figure 1.

\begin{tabular}{ccccccc}
\hline Symbol in fig. 1 & $T_{\text {fff }}(\mathrm{K})$ & $M\left(M_{\odot}\right)$ & $-\log M_{\mathrm{H}}$ & $-\log M_{\mathrm{He}}$ & $S(\mathrm{~s})$ & Modes $(\ell, k)$ \\
\hline Circles & $\mathbf{1 2 0 0 0}$ & $\mathbf{0 . 6 1 5}$ & $\mathbf{7}$ & $\mathbf{2}$ & 0.67 & $215.3(1,1), 275.0(1,2)$ \\
Triangles & 11500 & 0.75 & 5 & 2.5 & 0.97 & $\begin{array}{c}215.1(1,2), 265.1(1,3) \\
\text { Squares }\end{array}$ \\
& 12600 & 0.71 & 7.5 & 3 & 0.73 & $\begin{array}{c}215.4(1,1), 266.0(1,2) \\
301.6(1,3)\end{array}$ \\
Asterisks & 11500 & 0.85 & 8.5 & 3.5 & 0.19 & $\begin{array}{c}215.2(1,1), 271.5(1,2) \\
303.3(1,4)\end{array}$ \\
\hline
\end{tabular}

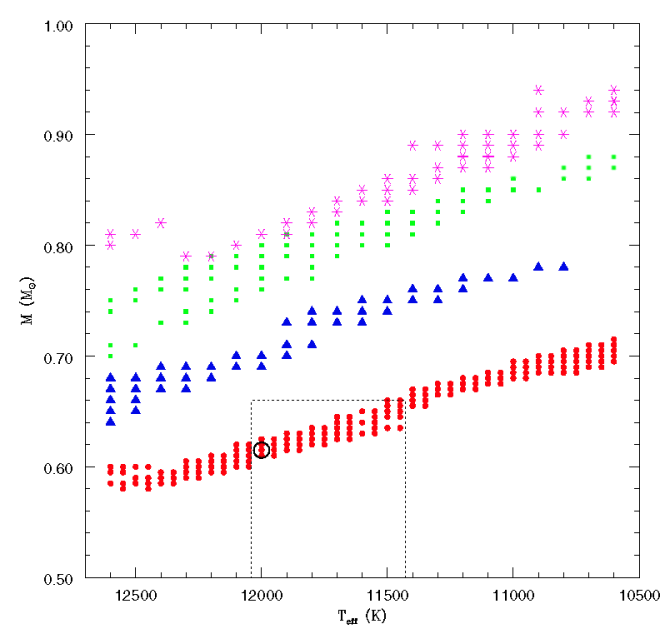

FIGURE 1. Results from comparison between the pulsation modes of the star G117-B15A and the models. The circles are the solutions for $M_{\mathrm{He}}=10^{-2} M_{*}$, the triangles for $M_{\mathrm{He}}=$ $10^{-2.5} M_{*}$, the squares for $M_{\mathrm{He}}=10^{-3} M_{*}$, and the asterisks for $M_{\mathrm{He}}=10^{-3.5} M_{*}$. The dotted line box limits the region of the independent temperature and mass determinations $( \pm 1 \sigma)$ and the open circle shows the location of the minimum for this family of solutions.

$\mathrm{C} / \mathrm{O}$ profile. Finally, he refines the adjustment even more and/or applies small changes to $M_{\mathrm{H}}, M_{\mathrm{He}}$, and in the core structure. He found that the mass of the $\mathrm{H}$ layer is either $M_{\mathrm{H}}=10^{-4} M_{*}$ or $10^{-7} M_{*}$, depending if the $215 \mathrm{~s} \ell=1$ mode is $k=2$ or 1 , respectively. His models were calculated basically from the same code as we use, although his version used the trace element approximation, while our updated version uses a parametrization that mimics the results of time-dependent diffusion calculations [1] to describe the transition zones.

The advantage of our approach is that we explore all possibilities, avoiding local minima and error propagation if the spectroscopic determinations are uncertain. Benvenuto et al. [2] used $\mathrm{He}, \mathrm{C}$, and $\mathrm{O}$ profiles calculated from previous stellar evolutionary phases, obtaining $T_{\mathrm{eff}}=11800 \mathrm{~K}, M=0.525 M_{\odot}$, and $M_{\mathrm{H}}=$
$10^{-3.83} M_{*}$, for G117-B15A. Their $T_{\text {eff }}$ and $M$ are consistent with UV spectrum fitting [24].

Our seismological study of G117-B15A is in agreement with other approaches, encouraging us to apply the same technique to other ZZ Ceti stars.

\section{SEISMOLOGY OF ZZ CETI STARS BY GROUPS}

The motivation of grouping the stars is to use the similarities to lower the uncertainties. For stars with similar temperature, mass, and excited modes, it is reasonable to expect that their internal structure would be similar as well. Our approach is akin to having several independent measurements for one particular star.

Our first task was the identification of all observed periodicities for ZZ Ceti stars. We re-analyzed the light curves of the stars observed at the $2.1 \mathrm{~m}$ telescope at McDonald Observatory for other projects [32, 33, 7, 34] and the new ZZ Ceti stars discovered with the $4.1 \mathrm{~m}$ telescope SOAR and the $1.6 \mathrm{~m}$ telescope at Observatório Pico dos Dias $[20,7]$. The other periods were obtained from the literature.

We divided the blue edge ZZ Ceti stars according to the excited mode with highest amplitude. We compared the observed modes with our model grid in the same way as for G117-B15A. We searched for common properties to characterize a particular group, as each group is a specific evolutionary stage in the white dwarf cooling.

$83 \mathrm{ZZ}$ Ceti stars have been selected from the SDSS sample. SDSS spectra for $g \leq 18$ stars have $\mathrm{S} / \mathrm{N} \sim 30$, and therefore, temperature and mass are reasonably well determined [21]. However, this is not the case for stars fainter than $g=18$. Bergeron et al. [3] showed that only with $\mathrm{S} / \mathrm{N} \geq 70$ one can obtain precise temperature $\left(\Delta T_{\text {eff }} \simeq 300 \mathrm{~K}\right)$ and gravity $(\Delta \log g \simeq 0.1)$. Their uncertainties are the external estimates, by fitting the $\mathrm{H}$ line profiles of duplicate spectra. The published uncertainties from SDSS spectra are the internal values, obtained by fitting the whole spectrum, combined with colors (see [10] for detailed explanation). The external uncertainties 
by comparing duplicated SDSS spectra are of order of $300 \mathrm{~K}[23,10,22]$. Kepler et al. [21], comparing SDSS and Gemini ( $\mathrm{S} / \mathrm{N} \geq 70$ ) spectra, found $\Delta T_{\mathrm{eff}} \simeq 320 \mathrm{~K}$, systematically lower in SDSS and $\Delta \log g \simeq 0.24 \mathrm{dex}$, systematically larger in SDSS.

Koester \& Allard [24] used UV spectra, which gives higher mass uncertainty, but much better temperature determination. An additional external uncertainty comes from the differences in the model grids from Kiel (e.g. [25]) and Montreal (e.g. [3]), which is around $200 \mathrm{~K}$.

We used the external temperature and mass determinations as a guide for the search of the best among all the possible families of seismological solutions.

\section{RESULTS AND DISCUSSIONS}

We have built an extensive model grid, calculating all modes that can be excited for a given internal structure at certain temperatures. We have also developed an independent technique of model fitting to compare the observed to the calculated periods, using the external temperature and mass determinations to guide the seismological solutions, never limiting the search to the uncertainty range of the spectroscopic determinations.

In our seismological study of ZZ Ceti stars, seismology proved to be a powerful tool in the study of stellar evolution. Even for stars with few excited modes, it is possible to determine some characteristic of their interior. For G226-29, with only one detected mode, it was possible to restrict the mass to be above $0.7 M_{\odot}$. Combined with reliable temperature and mass, three internal parameters could be determined.

The study of G185-32 was the motivation to include the relative amplitudes to give weights to the observed periods in the fits. The idea is that the high amplitude modes should be present in the best models. Our conclusion is that the observed amplitudes should be taken into account, even when one calculates the modes from an adiabatic model.

In the red edge, we studied HL Tau76 and G29-38. For the first one, we used the period spacing, because of the asymptotic behavior of the modes. Our analysis was consistent with the relative amplitudes between the $\ell=1$ and 2 modes. For G29-38, we suspected that the spectroscopic solutions were wrong, but seismology gives the same temperature and mass. It was a nice surprise that spectroscopy was right, agreeing with seismology, and that our prejudice that G29-38 should be in the red edge, was wrong.

We also studied BPM37093, a high mass white dwarf with $1.1 \pm 0.05 M_{\odot}$ (e.g., [3]), that has a significant crystallized portion. Even though it was not possible to determine the He layer mass, we could constrain the total stellar masses $\left(M>1 M_{\odot}\right)$, temperatures between 11600

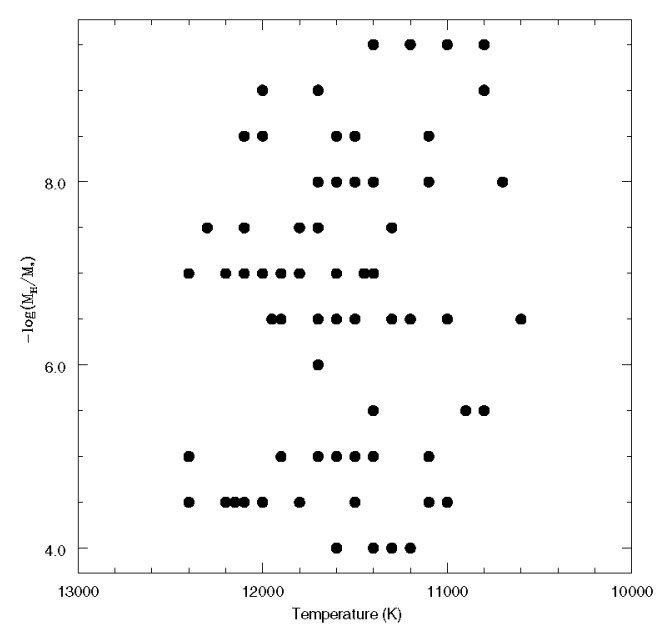

FIGURE 2. H mass vs. $T_{\mathrm{eff}}$, showing that there is no evidence of accretion nor loss of the external layers, as the ZZ Ceti stars evolve in the instability strip.

and $11800 \mathrm{~K}$, and $\mathrm{H}$ layer mass between $10^{-6} M_{*}$ and $10^{-7} M_{*}$, consistent with previous seismological determinations [1]. Our study of the high mass red edge is in agreement with theoretical predictions of temperatures higher than for low mass stars.

There are still many stars in the red edge that need to be re-observed with time-resolved photometry to detect more modes. This will allow us to study their interiors. We have demonstrated that it is possible to do seismology, even when a few modes are detected and/or reliable temperature and mass is available, with a minimum of four parameters.

Another important conclusion is that the $\mathrm{H}$ layer mass is not dependent on temperature (see Figure 2), according to Kolmogorov-Smirnov and correlation of coefficients tests. Therefore, there is no evidence for accretion or loss of the external layers, as it happens for Miras, as the $\mathrm{H}$ layer mass does not vary directly with temperature (or age). The mass loss of the external layer could come from the lack of reflectivity of the wave in the external layers [13].

The mean value for the $H$ layer mass is $10^{-6.3 \pm 1.6} M_{*}$, which is different than $10^{-4} M_{*}$, from evolutionary calculations. This indicates that some white dwarfs, even if their masses are near the most probable value, might have formed with $\mathrm{H}$ mass several orders of magnitude smaller than the value predicted by theory, i.e., it is probable that the mass loss during their evolution was more efficient than assumed by the models.

Using the seismological results, we derived an autoconsistent instability strip (see Figure 3 ), which includes the stars from the bright sample and the SDSS stars. This is the first time that the $\mathrm{ZZ}$ Ceti stars are studied seismo- 


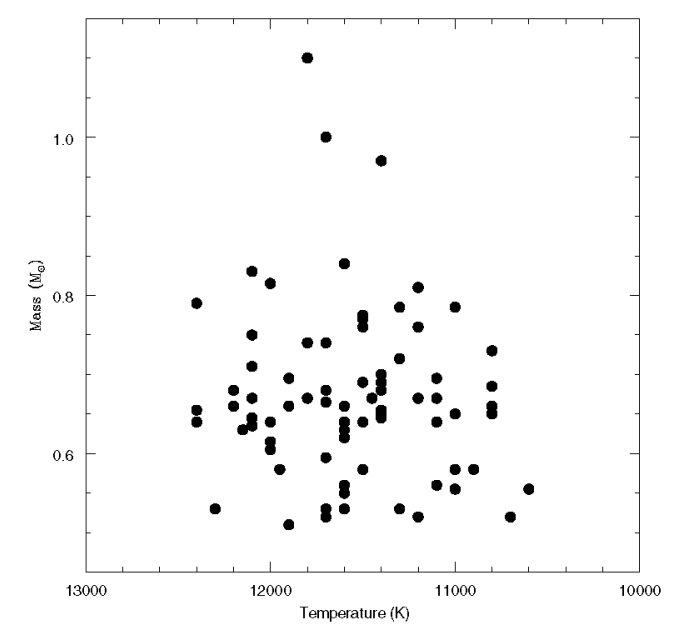

FIGURE 3. ZZ Ceti instability strip derived from seismology of these stars. We have included just the best solution for each studied star.

logically as a class. After 40 years since the discovery of the first ZZ Ceti star [29], we finally extracted information about this class as a whole.

\section{ACKNOWLEDGMENTS}

We acknowledge support from the CNPq-Brazil. We also acknowledge Travis S. Metcalfe for making available his scripts to calculate the model grid used in this work and Agnes Bischoff-Kim for sharing her version of the model with the Salaris profile. BGC thanks G. Fritz Benedict and the Stellar group at UT-Austin for the support to attend this meeting.

\section{REFERENCES}

1. Althaus, L. G., Serenelli, A. M., Córsico, A. H., \& Montgomery, M. H., A\&A 404, 593-609 (2003).

2. Benvenuto, O. G., Córsico, A. H., Althaus, L. G., \& Serenelli, A. M., MNRAS 332, 399-408 (2002).

3. Bergeron, P., Fontaine, G., Billères, et al., ApJ 600 , 404-408, (2004).

4. Bohm, K. H., \& Cassinelli, J., $A \& A$ 12, 21 (1971).

5. Bradley, P. A., ApJS 116, 307 (1998).

6. Brickhill, A. J., MNRAS 252, 334-341 (1991).

7. Castanheira, B. G., Kepler, S. O., Mullally, F., et al., $A \& A$ 450, 227-231 (2006).

8. Castanheira, B. G., Kepler, S. O., Costa, A. F. M., et al., A\&A 462, 989-993 (2007).

9. Castanheira, B. G., \& Kepler, S. O., MNRAS 385, 430-444 (2008).

10. Eisenstein, D. J., Liebert, J., Harris, H. C., et al., ApJS 167, 40-58 (2006)
11. Fontaine, G., Graboske, Jr., H. C., and van Horn, H. M., ApJS 35, 293 (1977).

12. Fontaine, G., Bergeron, P., Brassard, P. et al., ApJ 557, $792-797$ (2001).

13. Hansen, C. J., Winget, D. E., \& Kawaler, S. D., ApJ 297, 544-547 (1985).

14. Hine, B. P. A., "A search for pulsations in planetary nebulae nuclei" in Ph. D. Thesis, Texas Univ., Austin, 1988.

15. Iglesias, C. A., \& Rogers, F. J., ApJ 464, 943 (1996).

16. Itoh, N., Hayashi, H., Nishikawa, A., \& Kohyama, Y., ApJS 102, 411 (1996)

17. Kepler, S. O., Nather, R. E., McGraw, J. T., \& Robinson, E. L., ApJ 254, 676-682 (1982).

18. Kepler, S. O., Robinson, E. L., Koester, D., et al., ApJ 539 , 379-391 (2000).

19. Kepler, S. O., Costa, J. E. S., Castanheira, B. G., et al., ApJ 634, 1311-1318 (2005a).

20. Kepler, S. O., Castanheira, B. G., Saraiva, M. F. O., et al., $A \& A$ 442, 629-634 (2005b).

21. Kepler, S. O., Castanheira, B. G., Costa, A. F. M., \& Koester, D., MNRAS 372, 1799-1803 (2006).

22. Kepler, S. O., Kleinman, S. J., Nitta, A., et al., MNRAS 375, 1315-1324 (2007).

23. Kleinman, S. J., Harris, H. C., Eisenstein, D. J., et al., ApJ 607, 426-444 (2004).

24. Koester, D., \& Allard, N. F., Baltic Astronomy 9, 119-124 (2000).

25. Koester, D., Napiwotzki, R., Christlieb, N., et al., $A \& A$ 378, 556-568 (2001).

26. Kotak, R., van Kerkwijk, M. H., \& Clemens, J. C., $A \& A$ 413, 301-308 (2004).

27. Lamb, D. Q., ApJL 192, L129-L133 (1974)

28. Lamb, D. Q., \& van Horn, H. M., ApJ 200, 306-323 (1975).

29. Landolt, A. U., ApJ 153, 151 (1968).

30. McGraw, J. T., \& Robinson, E. L., ApJL 205, L155-L158 (1976).

31. Metcalfe, T. S., MNRAS 363, L86-L90 (2005).

32. Mukadam, A. S., Mullally, F., Nather, R. E., et al., ApJ 607, 982-998 (2004).

33. Mullally, F., Thompson, S. E., Castanheira, B. G. et al., ApJ 625, 966-972 (2005).

34. Mullally, F., Winget, D. E., Degennaro, S., et al., ApJ 676, 573-583 (2008).

35. Robinson, E. L., Mailoux, T. M., Zhang, E., et al., ApJ 438, 908-916 (1995).

36. Salaris, M., Dominguez, I., Garcia-Berro, E., et al., ApJ 486, 413 (1997).

37. Wegner, G., \& Reid, I. N., ApJ 375, 674-678 (1991).

38. Wood, M. A., "Astero-archaeology: Reading the galactic history recorded in the white dwarf stars" in Ph.D. Thesis, Texas Univ., Austin, 1990.

39. Zhang, E.-H., Robinson, E. L., \& Nather, R. E., ApJ 305 740-758 (1986). 